\title{
Medulloblastoma: molecular pathways and histopathological classification
}

Anna Borowska, Jarosław Jóźwiak

Department of Histology and Embryology, Center for Biostructure Research, Medical University of Warsaw, Warsaw, Poland

Submitted: 6 April 2014

Accepted: 5 August 2014

Arch Med Sci 2016; 12, 3: 659-666

DOI: 10.5114/aoms.2016.59939

Copyright $\odot 2016$ Termedia \& Banach

\section{Abstract}

Malignant brain tumors are the leading cause of cancer death among pediatric patients, and medulloblastoma constitutes $20 \%$ of them. Currently, the treatment is risk-adapted. Maximum surgical resection is recommended, always followed by chemotherapy and neuroaxis radiotherapy. In spite of the improving survival rate, survivors succumb to treatment-induced side effects. To reduce toxic effects, molecular-targeted treatment is proposed. Medulloblastoma research is very robust, and new articles on the subject are published daily. In the current review we have tried to bring together molecular pathophysiology of the neoplasm and current pathological classification, thus making an effort to relate tumor biology and the histological picture.

Key words: medulloblastoma, histopathology, molecular pathways.

Medulloblastoma $(\mathrm{MB})$ is one of the most common pediatric brain tumors [1]. Despite the hundreds of investigations conducted, the detailed molecular pathology of $\mathrm{MB}$ is still unknown. In a recently published paper, Taylor et al. divided MB into 4 molecular subgroups: Wnt, Sonic hedgehog, group 3 and group 4. Tumors are classified in each group on the basis of changes in the intracellular signaling [2]. The correlation between histological type, age, sex or prognosis is weaker. This article summarizes mutated pathways in individual histological subtypes of MB. It includes the molecular picture of classic $M B$, desmoplastic MB and large cell/anaplastic MB, having in mind clinical application of such data.

Novel molecular imaging techniques [3] should allow evaluation of the activity of e.g. kinases inside the patient's tumor, in vivo. In the near future, such techniques should permit precise determination of the status of selected molecular markers, without the need to perform surgery and take biopsy samples. When we know the triggers of the neoplastic process in a given tumor, we should be able to use specific inhibitors hindering activation of molecular pathways involved in tumor development, e.g. vismodegib (for Sonic hedgehog), sirolimus (for tyrosine kinases) or OMP-18R5 and XAV939 (for the Wnt pathway), or even use them in combination in cases where several pathways are hyperactive. Also activation of endogenous molecules cold be used for this purpose [4].

Brain tumors, after leukemia, are the major cause of death in pediatric oncology. The most common tumor, constituting $20 \%$ of all pediatric brain tumors, is $\mathrm{MB}$, a small blue-cell malignant tumor which arises in

\author{
Corresponding author: \\ Jarosław Jóźwiak \\ Department of Histology \\ and Embryology \\ Center for Biostructure \\ Research \\ Medical University of Warsaw \\ 5 Chalubinskiego St \\ 02-004 Warsaw, Poland \\ E-mail: jaroslaw.jozwiak@ \\ gmail.com
}


the posterior cranial fossa and in the cerebellum, mainly in the hemispheres and the vermis $[2,5$, 6]. All variants of $M B$ arise from primitive neuroectodermal cells [1]. Patient symptoms result from alterations in the cerebellum (e.g. ataxia, hypotonia) and increased intracranial pressure (e.g. headache, vomiting without nausea, ocular palsies). Metastases spread via cerebrospinal fluid (CSF), rarely localizing outside the central nervous system (CNS) [1]. According to the new WHO classification (2007), medulloblastoma is divided into five groups: classis type (CMB), desmoplastic/nodular type (DN), medulloblastoma with extensive nodularity (MBEN), anaplastic type and large cell medulloblastoma (LC) [7]. As anaplastic medulloblastoma and large cell medulloblastoma often appear together, sometimes they are named large cell/anaplastic medulloblastoma (LC/A) [7]. Because of the similar histological pattern, DN and MBEN are classified as desmoplastic medulloblastoma (DMB).

Risk stratification is based on patient's age, metastasis, extent of resected tissue and anaplasia in histology [1]. Patients over 3 years with localized lesions, no anaplasia, no metastases and residual tumor tissue below $1.5 \mathrm{~cm}^{2}$ have good predicted outcome and are classified as the standard risk group. The high-risk group with poor outcome prognosis consists of very young children with dispersed metastases and unresectable tumor or residual tumor after surgery over $1.5 \mathrm{~cm}^{2}$ [1]. Currently, the treatment is risk-adapted. Maximum surgical resection is recommended, always fol-

Without Wnt signal
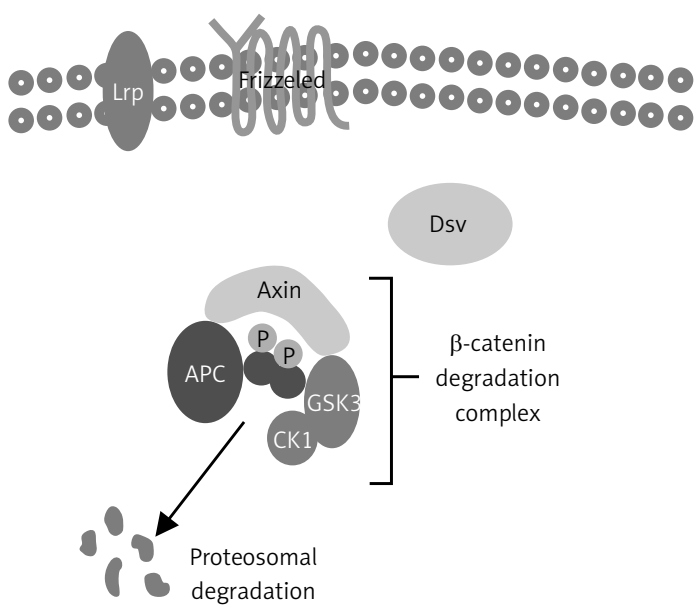

Figure 1. Wnt pathway in active and inactive state lowed by chemotherapy and neuroaxis radiotherapy [1]. The treatment of MB causes serious consequences for the developing CNS. Thus, patient stratification should include progression risk and aggressiveness of the disease and, consequently, the type and intensity of therapy. This underlines the importance of molecular research which leads to MB subtype identification.

In a recently published paper, $M B$ has been divided into 4 molecular subgroups: Wnt (a combination of Wg (wingless) and Int (integration)) subgroup, Sonic hedgehog subgroup, group 3 and group 4 [2].

The Wnt/ $\beta$-catenin pathway participates in the control of vertebrate development [2] (Figure 1). In the absence of the Wnt molecule, $\beta$-catenin is targeted to degradation by the APC/Axin/GSK-3 $\beta$ complex and phosphorylated. This leads to proteolysis of $\beta$-catenin in the proteasome. If Wnt binds to its receptor Frizzled and the Lrp5 or Lrp6 co-receptor, Disheveled (Dsv) is activated. Active Dsv inhibits the $\beta$-catenin degradation complex. $\beta$-catenin translocates to the nucleus, where it promotes transcription of target genes [5].

The Wnt subgroup includes mainly classic $M B$, but LC/A tumors with a good prognosis are present here too [2]. Patients with Turcot syndrome are a separate group, with a predisposition to Wnt MB. The mechanism underlying this disease is germline mutation of $A p c[2,5]$. The product of $A p c$ inhibits the Wnt pathway. Furthermore, a somatic mutation in Ctnnb1 (encoding $\beta$-caten-

With Wnt signal

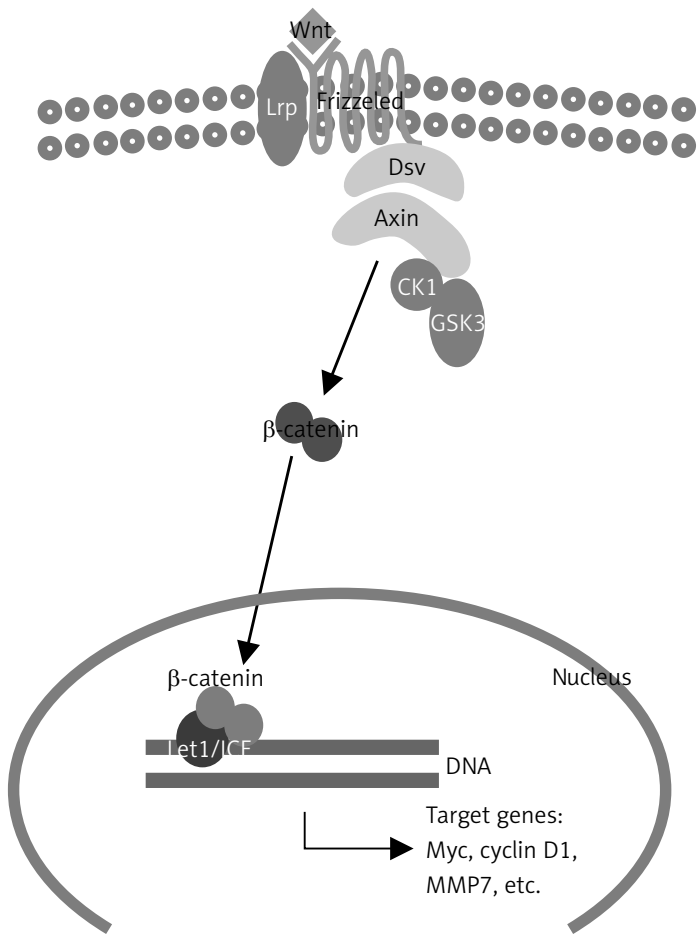


in) has been described in sporadic $M B$ [2]. A less common mutation in AXIN1 and deletion of AXIN2 have been described. The monosomy of chromosome 6 is present in about 100\% of Wnt tumors $[2,5]$. The Wnt MBs can occur at any age, with equal frequency in both sexes. They are rare among children below 3 years. The long-term survival prognosis exceeds $90 \%$ [2].

The Sonic hedgehog subgroup is named after the Sonic hedgehog (Shh) signaling pathway (Figure 2). Hedgehog proteins, as well as Wnt, are active in the development of vertebrate tissues [1]. Activation of the Shh pathway is mediated by translocation of Smoothened (SMO, encoded by Smo) to the cell membrane. Thus Gli (the glioblastoma family protein), a Shh signaling effector, is relieved from kinase suppression and can translocate to the nucleus, to activate hedgehog target genes. In the absence of Shh, the transmembrane protein Patched (encoded by Ptch) blocks Smoothened activation by maintaining its sequestration.

Germline mutations in Ptch1, which are characteristic for patients with Gorlin syndrome, predispose to Shh-subgroup MB [1]. Other molecular abnormalities found in this subgroup are somatic mutations in Ptch, Sufu and Smo, as well as amplification of Gli1 and Gli2 [8]. Sufu encodes the suppressor of fused homolog (SUFU), a component of the Shh pathway, which interacts with Gli1 [5]. As in the case of the Wnt subgroup, the gender ratio is $1: 1$. These tumors occur predominantly in two age groups - infants ( $0-3$ years) and adults (over 16 years). The prognosis varies depending on the age and appears to be good in infants and moderate in older children. Shh group tumors show a high incidence in desmoplastic/nodular, rarely classic or LC/A histopathology [2]. In the majority of Shh MBs, the Shh pathway is co-activated with CXCR4 $[8,9]$.

In the case of groups 3 and 4, MBs have not been directly associated with a particular signaling pathway. The high level of Myc expression characteristic for group 3 is mostly due to Myc amplification. Groups 3 and 4 demonstrate amplification and overexpression of OTX2, a transcription factor participating in brain development. The majority of group 3 tumors histologically belong to $C M B$, but the frequency of LC/A is higher than in other groups. These MBs occur usually between 0 and 16 years, are more common in males, and are almost never seen in adults. Owing to frequent metastases, the prognosis is poor. The pathogenesis of group 4 is not completely understood. The only molecular $a b-$ normality specific for this group is loss of chromosome $X$, which is not present in all cases. However, the most common change, the presence of isochromosome $17 q$, is the same for both groups [2].

As yet, there are no markers that could become a gold standard in the diagnosis of each type of MB. Nevertheless, the specific histology of neoplasm could indicate the molecular profile (Table I). Apart from the previously mentioned Wnt and Shh pathways, increased activity of hormone- and growth factor-activated pathways needs highlighting. Akt/PKB (protein kinase B) and Erk-1/2 (extracellular-signal-regulated kinase-1/2, also known as MAPK3 and MAPK1) regulate cell proliferation by preventing inhibitory activity of TSC (tuberous sclerosis proteins) on mTOR (mammalian target of rapamycin) (Figure 3). mTOR is a component of two complexes - mTOR Complex 1 (mTORC1) and mTOR Complex 2 (mTOR2) [6, 10]. mTORC1 plays a critical role in tumorigenesis [10]. One of its nu-
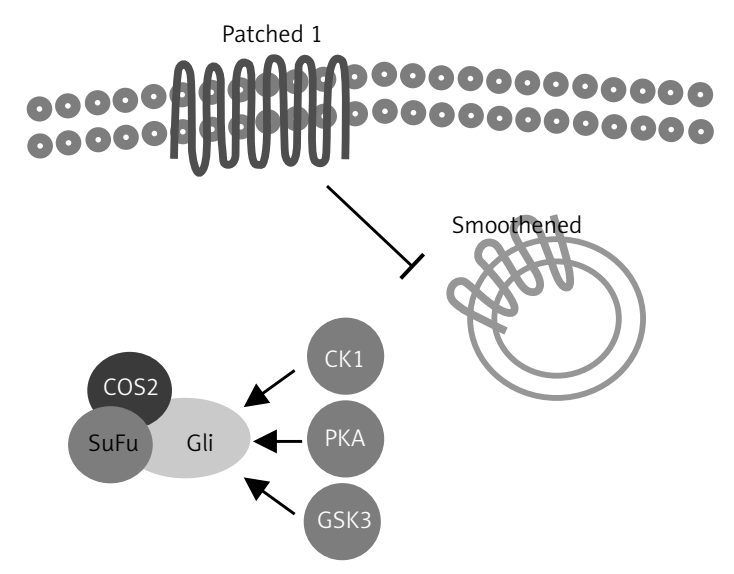

Figure 2. Activation of the Shh pathway

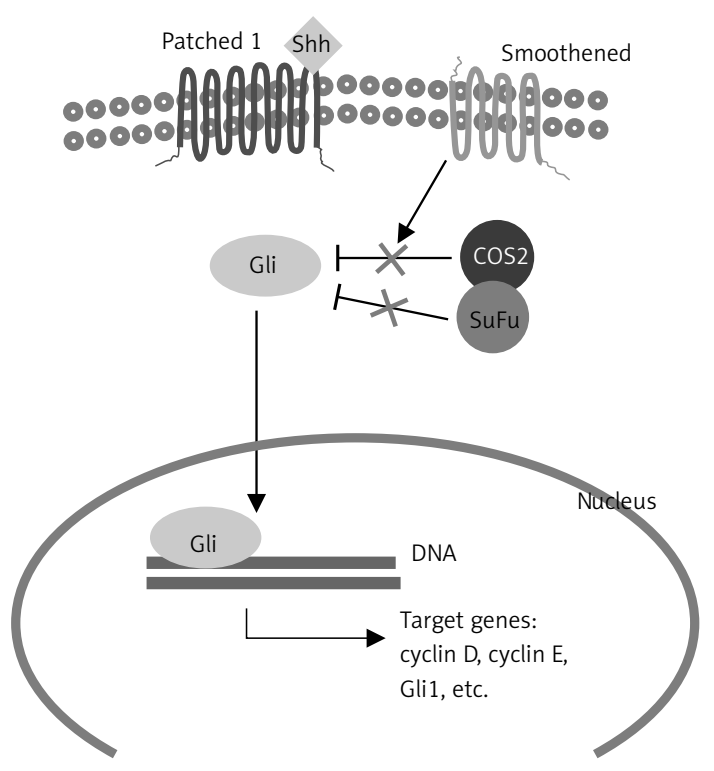


Table I. Current classification of molecular subgroups of medulloblastoma

\begin{tabular}{|lccc|}
\hline Group & Type & Molecular changes & Prognosis \\
\hline Wnt & Classic & Ctnnb1, Apc & Good \\
\hline Sonic hedgehog & DN & Ptch1, Sufu, Smo & Good/intermediate \\
\hline 3 & Classic, LC/A & Myc & Poor \\
\hline 4 & Classic, LC/A & $?$ & Intermediate \\
\hline
\end{tabular}

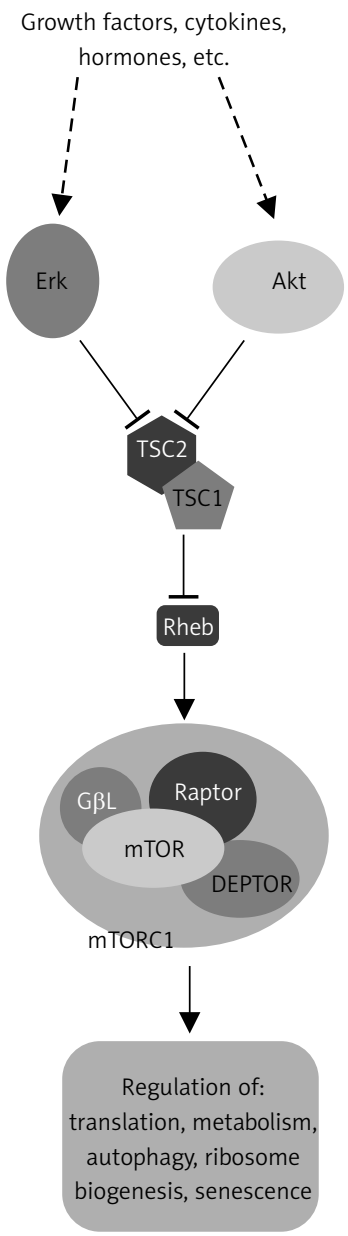

Figure 3. Importance of the Akt/PKB, Erk and mTor pathways for cell proliferation

merous biological effects is stimulation of translation of mRNA encoding C-Myc, N-Myc and cyclin D1 [6]. mTORC1 is composed of mTOR, regulatory-associated protein of mTOR (Raptor), mammalian LST8/G-protein $\beta$-subunit like protein ( $\mathrm{mLST} / \mathrm{G} \beta \mathrm{L}$ ), PRAS40 (proline-rich Akt/PKB substrate $40 \mathrm{kDa}$ ) and DEPTOR (DEP domain-containing mTOR-interacting protein). mTORC1 exerts its regulatory effect by phosphorylation of ribosomal S6 kinase (S6K1) and $4 \mathrm{E}-$ binding protein (4E-BP1) [10]. It is suggested that the TSC complex may bind to the $\beta$-catenin degradation complex and simultaneously participate in Wnt signaling, inhibiting gene transcription [11]. Molecular classification has not been correlated with each histological type of MB yet (Table II).

\section{Classic medulloblastoma}

Classic MB (CMB) is characterized by sheets of pleomorphic, small, round or oval cells, usually with a high nuclear : cytoplasmic ratio. HomerWright rosettes indicating neuroblastic differentiation are occasionally found in some cases. Such tumors have two peak ages of onset, 10 years and 20 years, and rarely occur in children under 3 years. The prognosis is good. Classic type is described in around $70 \%$ of cases of MB [5]. It usually arises in the cerebellar vermis. Classic MB may evolve into anaplastic MB [7].

Tumors with classic histology usually exhibit alterations in the Wnt pathway, C-Myc and N-Myc mediated signaling, the Shh pathway and pathways activated by insulin-like growth factor receptor I [2, 12, 13]. The classic type has been found in all subgroups described by Taylor et al. and was the only type showing alterations in the Wnt pathway. Mutations in Wnt pathway components are found in the majority of classic MBs and seem to be favorable prognostic factors [2]. The previously mentioned Turcot syndrome predisposes to CMB. In contrast to the close correlation between germline mutations and APC, somatic mutations in this gene are rare [5]. Mutations in axin-1 are not frequent either. The most common alteration in Wnt signaling is Ctnnb1 mutation. Changes in the primary structure of $\beta$-catenin cause insensitivity of the protein to phosphorylation by GSK3 and consequently its uncontrolled upregulation. $\beta$-catenin nuclear positivity is mostly linked to monosomy of chromosome 6 [2]. Moreover, the Wnt pathway may be enhanced by the SOX (Sry-related HMG box) family. Sox4 and Sox 11 undergo overexpression in CMB and lead to hyperactivity of the Wnt pathway by binding $\beta$-catenin and Lef/TCF, and by stabilizing $\beta$-catenin [14]. Despite the fact that $M y c$ and $\mathrm{N}$-Myc amplification is a poor prognosis indicator, individuals with strong activity of the Wnt pathway are ranked in the low-risk group [15]. However, $\mathrm{N}-\mathrm{Myc}$ amplification in Shh tumors has been associated with better prognosis than $N-M y c$ amplification in non-Shh tumors [13]. Protein 53 (p53, encoded by TP53) has been associated with a dismal prognosis in children with MB [16]. Wnt $M B$ with mutated $p 53$ has a more favorable prognosis [17]. Overexpression of $\beta$-catenin causes 
Table II. Molecular pathways implicated in the development of three distinctive forms of MB: classic medulloblastoma (CMB), dysplastic medulloblastoma (DMB) and large-cell and anaplastic medulloblastoma (LC/A MB)

\begin{tabular}{|lccc|}
\hline Variable & CMB & DMB & LC/A MB \\
\hline Wnt pathway & $+[2,5]$ & $-[1,2,5]$ & $+[2]$ \\
\hline Shh pathway & $+[2,12]$ & $+[1,2,5,8,12]$ & $+[2,13]$ \\
\hline Myc & $+[2,13]$ & $+[2,14]$ & $+[1,2,13,15,25]$ \\
\hline CXCR4 & $+[9]$ & $+[5,8,9]$ & $+[9]$ \\
\hline IGFs & $+[19]$ & $+[19,20]$ & nd \\
\hline Erk & $+[11]$ & $+[28]$ & nd \\
\hline Akt & $+[11]$ & $+[28]$ & $+[22]$ \\
\hline OTX1, OTX2 & $+[5,21,22,23]$ & $+[6,21]$ & $+[18,30]$ \\
\hline p53 & $+[17,18]$ & $+[8,16]$ & $+[1,32]$ \\
\hline erbB-2 & $+[1,32]$ & $+[1,32]$ & \\
\hline
\end{tabular}

+ pathway implicated in the development of tumor, - pathway not found to be implicated in the development of tumor, nd - no data.

accumulation of transcriptionally active p53, and although an adverse influence on patient survival can be expected, concomitant mutation of Ctnnb1 and TP53 is a predictor of long-term survival [18].

The Shh CMBs display a worse prognosis than Shh desmoplastic tumors [12], while it seems that the Shh pathway is co-activated with the CXCR4 pathway [8]. However, the CXCR4 level is higher in DMB than in classic MB [9].

Insulin-like growth factors (IGFs) are proteins with high sequence similarity to insulin. The IGFs are part of a complex system which consists of IGF-I (IGF-1) and IGF-II (IGF-2), two receptors, IGFIR and IGF-IIR, and six specific IGF-binding proteins (IGFBP1-IGFBP6). The IGF-1 participates mainly in adult metabolism, whereas IGF-2 regulates development of organs in the fetal state [6]. The IGF-IR is highly expressed during embryonal and early postnatal development and becomes lower in adolescence. IGF-IR belongs to the tyrosine kinase receptor family (receptor tyrosine kinases, RTK); it transduces the signal through its intercellular tyrosine kinase linked to the RAS/RAF/MAPK pathway (MAPK/Erk pathway) and PI3K/Akt pathway [6]. The MBs show enhanced IGF expression [19, 20]. Moreover, due to IGF-1 stimulation, not only is IGF-IR continuously activated, but also IRS-1, Akt and Erk-1/2 are phosphorylated [19]. Both Erk1/2 and Akt abolish control over mTOR, which initiates translation, protein synthesis and cell mass gain [10]. Akt seems to participate in Shh CMB pathogenesis [6]. In MAPK/Erk signaling, the growth factor binds to the RTK and leads to phosphorylation of a GTPase named Ras. Active Ras binds to Raf, a serine/tyrosine protein kinase which phosphorylates and activates MEK (mitogen-activated protein kinase kinase), which in turn activates Erk-1 and Erk-2 through phosphorylation. Downstream targets of the Erk signaling pathway regulate transcription and translation [6, 11, 12]. Recent data suggest upregulation of the Erk pathway in $M B$. It has been shown that some CMBs exhibit an elevated level of Raf, MEK and consequently Erk. Additionally, as previously mentioned, the Wnt pathway may interact with the mTOR inhibitor-TSC complex and inhibit gene transcription. In Erk-positive tumors, the TSC complex is arrested, and Wnt pathway target genes are transcribed. As $\beta$-catenin is a transcription factor for such genes as cyclin D, C-Myc and N-Myc, its increased level may predict tumor progression [11].

Classic MB express calbindin, specific for the cells in the cerebellar ventricular zone. Thus, it is suggested that these tumors originate from cells in the ventricular matrix and Purkinje neurons, and arise more commonly in the cerebellar vermis. However, CMBs negative for calbindin develop rarely from the external granular layer (EGL). Both calbindin-positive and calbindin-negative MBs show an increased level of OTX2. OTX2 and OTX1 are transcription factors belonging to the bicoid subfamily which may play a role in brain development [21]. There is no association between OTX2 level and metastasis, prognosis or tumor recurrence, but OTX2-positive tumors were mainly obtained from children between 5 and 20 years old. OTX2 expression determines the correlation with classic histology [21, 22]. Additionally, OTX2-positive CMB may express OTX1 as well [21]. OTX2-positive tumors express neither Shh pathway nor Wnt pathway components [23].

\section{Desmoplastic medulloblastoma}

In view of similar histology, MBEN and DN are grouped together as desmoplastic MB. This type 
features nodules scattered among internodular spaces consisting of undifferentiated cells and regions of desmoplasia. Nodules are composed of differentiated neurocytic cells. The MBEN (about $20 \%$ of desmoplastic $M B$ ) is characterized by a high number of irregularly shaped nodules, whereas DN $M B$ nodules are sparse, but dispersed in vast regions of desmoplasia [5, 7]. DN type can be subdivided into conventional DN MB (about 67\%) and paucinodular DN MB [5]. Desmoplastic MB arises in cerebellar hemispheres, usually in two age groups: children below 3 years and adults [2, 24]. MBEN is reported only in young children and seems to be the end stage of DN differentiation. Prognosis is better than for the classic subtype, and consequently $D M B$ is classified in the low-risk group [7]. Although the majority of DMBs predict a favorable clinical outcome, desmoplastic tumors with aggressive behavior are also present in this subgroup [16].

It has been suggested that all DMBs harbor alterations in the Shh pathway $[2,5,8]$. The most frequent mechanism of constant Shh pathway activation involves mutations in Patched1 (due to loss of heterozygosity on chromosome 9q), rarely Smo or Sufu $[2,5]$. Germline mutation of Sufu predisposes to $M B$ in progeny, but the penetrance is incomplete [25]. Hedgehog target genes can be upregulated by activation of Notch family members [6]. The level of growth-promoting Notch 2 is elevated in some cases of MB, especially those with activation of Shh signaling and crosstalk with the Wnt pathway. Because of this correlation, overexpression of Notch 2 is most probable in desmoplastic or classic MBs. Upregulated levels of Notch 2 and its downstream target factor Hes 1 indicate a poor prognosis and predict shorter survival [6]. Deregulation in Shh signaling leads to overexpression of transcription factors such as Gli1, and also the Myc family [8]. N-Myc is a member of the Myc family of transcription factors, regulated by Shh induced in desmoplastic tumors. Tumors associated with Shh and N-Myc are usually less aggressive DN MB [13]. N-Myc is required for tumorigenesis, so its inhibition plays a crucial role in the prevention of tumor growth. Neurotrophic tyrosine kinase receptor type 3 (TrkC) is a predictor of longer survival [1]. TrkC is a catalytic receptor for neurotrophin 3 and mediates multiple effects, including apoptosis and neuronal differentiation, in MB. The high TrkC mRNA level in intranodular regions of DMBs could explain the favorable clinical outcome of patients with such a tumor [25].

Desmoplastic MB with aggressive clinical behavior may harbor p53 over-expression [16]. Patients with Li-Fraumeni syndrome, a hereditary disorder caused by heterozygous germline mutation in p53, suffer from bone and soft-tissue sarcomas, premenopausal breast carcinoma, brain tumors, adrenocortical carcinoma, and leuke- mias [26]. MB in patients with Li-Fraumeni syndrome is another example of Shh MB with poor prognosis [16].

As DMBs arise usually in the cerebellar hemispheres, they could originate from progenitor cells in the external granular layer (EGL) [6]. EGL precursor cell markers, such as ATOH1 (atonal homolog 1), CXCR4 (C-X-C chemokine receptor type 4) and P75 ${ }^{\text {NTR }}$, are found in the DMB [27]. CXCR4 is a chemokine receptor specific for stromal cell-derived factor 1 (SDF-1) chemokine, participating in brain development. A high level of CXCR4 is usually present in $\mathrm{DMB}$ or $\mathrm{CMB}$ occurring in the youngest patients $[8,9]$. CXCR4 is overexpressed in the DMB Shh subtype. CXCR4 upregulation seems to be the result of Gli1 function. The Shh pathway influences the cell surface level of CXCR4, whereas CXCR4 regulates some of the Shh target genes, e.g. cyclin D1. Additionally, CXCR4 maximizes Shh tumor proliferation [9]. p75 ${ }^{\text {NTR }}$, also known as the low-affinity nerve growth factor receptor (NGFR), contains an intercellular death domain. Since p75 ${ }^{\mathrm{NTR}}$ expression is increased in DMB (in contrast to $\mathrm{CMB}$ ), this protein stimulates apoptosis in $\mathrm{DMB}$ and might be a suppressor factor which leads to a good prognosis [27].

Although OTX1 and OTX2 are detectable in CMB, tumors with sole expression of OTX1 are mainly localized in the hemispheres and display desmoplastic/nodular histology. Scarce OTX2 presence in $\mathrm{DN} M B$ is restricted to poorly differentiated internodular regions [21].

mTOR and its upstream kinases, Erk-1/2 and Akt/PKB, seem to be good prognosis factors in a special DMB type, MBEN [28]. The Akt/PKB pathway may be activated by many RTKs, such as PDGFR, IGF-IR or EGFR. An extracellular signaling molecule, for example IGF-II, binds to its receptor and activates phosphatidylinositol-3-kinase (PI3K), which phosphorylates PIP2 (phosphatidylinositol $(3,4)$-bisphosphate) to PIP3 (phosphatidylinositol (3,4,5)-trisphosphate). Then PIP3, in cooperation with PDK-1 (3-phosphoinositide-dependent kinase 1), phosphorylates Akt/PKB, which influences cell survival (via $\mathrm{Bcl}-2$, an antiapoptotic agent), cell growth and proliferation (via the mTOR complex), angiogenesis (through eNOS), cellular metabolism (through stimulation of glucose uptake in response to insulin) and cell migration and invasion [29]. IGF-II expression status correlates with desmoplastic subtype with the upregulated Shh pathway [19]. Research conducted on murine models revealed that IGF-II is indispensable for tumor progression [6]. IGF-II has proliferative effects on MB cells. It also induces phosphorylation of Akt protein and its downstream target

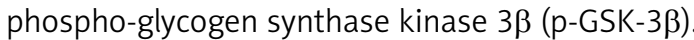
Additionally, Shh DMB co-stimulated with IGF-II demonstrates an increase in cyclin D1 and Gli1 
expression [20]. The research conducted by our group showed the implication of S6K1 and 4E-BP1, mTORC1 effectors [28]. Due to the interplay between mTORC1 and Akt, the Akt/PKB pathway components are upregulated, too [10]. PDK-1 as well as Akt has been found to be hyperactivated in MBEN. Moreover, the level of $p$-GSK-3 $\beta$ can be increased. Another mTOR activator, the MAPK/ERK pathway, may be triggered, as elevated levels of MEK and Erk have been detected in these tumors [28].

\section{Large cell and anaplastic medulloblastoma}

Large cell and anaplastic medulloblastoma (LC/A) subtype accounts for about $20 \%$ of diagnosed MBs [5]. These tumors, known for their aggressive behavior, tend to metastasize via CSF and outside the CNS [1]. However, LC/A MB might have a good prognosis if clinical risk factors, such as metastasis and young patient age, do not occur. Still, LC/A pathology is a high-risk factor (as well as metastases at presentation or high-frequency Myc amplification) [15].

LC MB is characterized by large, round cells with giant nuclei and prominent nucleoli, while anaplastic MB is characterized by undifferentiated cells with pleomorphic, angular nuclei $[5,7]$.

The Myc family comprises C-Myc, N-Myc and L-Myc. C-Myc protein is a transcription factor which modulates various cell functions, for example cell proliferation. Myc can be activated by the Shh or Wnt signaling pathway. It is also the most commonly amplified proto-oncogene in $\mathrm{MB}$ [15]. Myc or N-Myc together with LC/A histology is a poor prognosis factor $[5,15,26]$. An elevated level of C-Myc is associated with LC/A MB subtype. Increased expression of this proto-oncogene is the result of amplification, copy number gain or Wnt subgroup classification; however, C-Myc amplification is the most common for LC/A tumors [15]. Additionally, C-Myc mRNA expression is associated with anaplasia and predicts shorter patient survival. Many anaplastic tumors demonstrate $\mathrm{N}$-Myc overexpression, but there is no substantial association with anaplasia [25]. Recent studies have shown that N-Myc amplification may have no prognostic relevance, due to high incidence of alterations within the Shh pathway - a good prognostic factor [13].

The TP53-ARF (alternate reading frame) pathway is altered in the majority of human cancers. ARF is a cell inhibitor which indirectly stabilizes p53, which leads to growth arrest and apoptosis [1]. In LC/A MB changes may be related to p53 mutations or ARF alterations. A high level of p53 is a poor clinical factor in MB in general. Nevertheless, alteration in p53 or ARF seems to act as a significant factor in LC/A MB development [30]. Fran et al. are currently investigating whether concomi- tant TP53-ARF modification and Myc amplification play a role in LC/A tumor development $[18,30]$.

Anaplastic MB tends to express glycoproteins from the receptor tyrosine kinase family I, also known as the ErbB or EGF receptor family [6]. The ErbB family consists of 4 members: erbB-1 (epidermal growth factor- EGFR), erbB-2 (Her2/neu), erbB-3 and erbB-4. Two receptors, erbB-2 and erbB-4, play an important role in $M B$ pathogenesis [31]. ErbB-2-positive tumors are more likely to present aggressive behavior [1, 32]. Dysregulated expression of this receptor is found in all histological subtypes; however, the highest level has been observed in LCA subtypes [32]. ErbB-2, via the PI3K/Akt pathway and Erk1/2 pathway, induces expression of a prometastatic gene, S100A4. Thus, overexpression of erbB-2 correlates with metastasis [33]. The Shh pathway probably downregulates erbB-4 expression [20]. MB cells, which exhibit a high Gli1 level, at the same time present normal or low erbB-4. Hence, tumors with low Gli1 demonstrate high erbB-4. This correlation is maintained in the case of the CYT1 erbB-4 isoform, being probably the only isoform of erbB-4 which is capable of activating PI3K/Akt signaling. Through this pathway, the antiapoptotic signal is transmitted [1]. ErbB-2 and erbB-4 co-expression together with the anaplastic histotype is a poor prognostic factor [1, 31, 32].

Otx2 plays a significant role in the presence of anaplasia. Amplification of Otx2 is a predictor of poor prognosis and dismal clinical outcome. Furthermore, OTX2 regulates Myc expression [22].

\section{Summary}

In the current paper we have reviewed molecular pathways implicated in MB development, trying to prepare the grounds for in vivo molecular imaging that should allow determination of the status of the pathways identified in basic research. We hope that specific kinase inhibitors and other molecules that would be able penetrate the bloodbrain barrier and inhibit those pathways, some of which are currently in clinical trials or have already been registered in the treatment of brain tumors (vismodegib, sirolimus), will soon bring a positive breakthrough in this patient population.

\section{Conflict of interest}

The authors declare no conflict of interest.

\section{References}

1. Gilbertson RJ. Medulloblastoma: signalling change in treatment. Lancet Oncol 2004; 5: 209-18.

2. Taylor MD, Northcott PA, Korshunov A, et al. Molecular subgroups of medulloblastoma: the current consensus. Acta Neuropathol 2012; 123: 465-72. 
3. Schaller BJ, Cornelius JF, Sandu N, Buchfelder M. Molecular imaging of brain tumors personal experience and review of the literature. Curr Mol Med 2008; 8: 711-26.

4. Ma J, Sawai H, Matsuo Y, et al. IGF-1 and PTEN regulate the proliferation and invasiveness of colon cancer cells through opposite effects on PI3K/Akt signalling. Arch Med Sci 2009; 5: 195-206.

5. Ellison DW. Childhood medulloblastoma: novel approaches to the classification of a heterogeneous disease. Acta Neuropathol 2010; 120: 305-16.

6. Jóźwiak J, Grajkowska W, Włodarski P. Pathogenesis of medulloblastoma and current treatment outlook. Med Res Rev 2007; 27: 869-90.

7. Louis DN, Ohgaki H, Wiestler OD, et al. The 2007 WHO classification of tumors of the central nervous system. Acta Neuropathol 2007; 114: 97-109.

8. Yoon JW, Gilbertson R, Iannaccone S, Iannaccone P, Walterhouse D. Defining a role for Sonic hedgehog pathway activation in desmoplastic medulloblastoma by identifying GLI1 target genes. Int J Cancer 2009; 124: 109-19.

9. Sengupta R, Dubuc A, Ward S, et al. CXCR4 activation defines a new subgroup of sonic Hedgehog - driven medulloblastoma. Cancer Res 2012; 72: 122-32.

10. Efeyan A, Sabatini DM. mTOR and cancer: many loops in one pathway. Curr Opin Cell Biol 2010; 22: 169-76.

11. Wlodarski PK, Boszczyk A, Grajkowska W, Roszkowski $M$, Jozwiak J. Implication of active Erk in the classic type of human medulloblastoma. Folia Neuropathol 2008; 46: 117-22.

12. Northcott PA, Hielscher T, Dubuc A, et al. Pediatric and adult sonic hedgehog medulloblastomas are clinically and molecularly distinct. Acta Neuropathol 2011; 122 : 231-40.

13. Korshunov A, Remke $M$, Kool $M$, et al. Biological and clinical heterogeneity of MYCN-amplified medulloblastoma. Acta Neuropathol 2012; 123: 515-27.

14. de Bont JM, Kros JM, Passier MM, et al. Differential expression and prognostic significance of SOX analysis. Neuro Oncol 2008; 10: 648-60.

15. Ryan SL, Schwalbe EC, Cole M, et al. MYC family amplification and clinical risk-factors interact to predict an extremely poor prognosis in childhood medulloblastoma. Acta Neuropathol 2012; 123: 501-13.

16. Gessi M, von Bueren AO, Rutkowski S, Pietsch T. p53 expression predicts dismal outcome for medulloblastoma patients with metastatic disease. J Neurooncol 2012; 106: $135-41$

17. Lindsey JC, Hill RM, Megahed $\mathrm{H}$, et al. TP53 Mutations in favorable-risk Wnt/wingless-subtype medulloblastomas. J Clin Oncol 2011; 29: e344-6.

18. Pfaff E, Remke M, Sturm D, et al. TP53 mutation is frequently associated with CTNNB1 mutation or MYCN amplification and is compatible with long-term survival in medulloblastoma. J Clin Oncol 2010; 28: 5188-96.

19. Hartmann W, Koch A, Brune $\mathrm{H}$, et al. Insulin-like growth factor II is involved in the proliferation control of medulloblastoma and its cerebellar precursor cells. Am J Pathol 2005; 166: 1153-62.

20. Corcoran RB, Bachar Raveh T, Barakat MT, Lee EY, Scott MP. Insulin-like growth factor 2 is required for progression to advanced medulloblastoma in patched 1 heterozygous mice. Cancer Res 2008; 68: 8788-95.

21. de Haas T, Oussoren E, Grajkowska W, et al. OTX1 and OTX2 expression correlates with the clinicopathologic classification of medulloblastomas. J Neuropathol Exp Neurol 2006; 65: 176-86.
22. Adamson DC, Shi O, Worhtam M, et al. OTX2 is critical for the maintenance and progression of classic medulloblastoma. Cancer Res 2010; 70: 181-91.

23. Adamson DC, Shi Q, Wortham M, et al. OTX2 is critical for the maintenance and progression of Shh-independent medulloblastoma. Cancer Res 2010; 70: 181-91.

24. Brugieres L, Pierron G, Chompret A, et al. Incomplete penetrance of the predisposition to medulloblastoma associated with germ-line SUFU mutations. J Med Genet 2010; 47: 142-4.

25. Eberhart CG, Kratz J, Wang Y, et al. Histopathological and molecular prognostic markers in medulloblastoma: c-myc, N-myc, TrkC, and anaplasia. J Neuropathol Exp Neurol 2004; 63: 5.

26. Li FP, Fraumeni JF Jr. Soft-tissue sarcomas, breast cancer, and other neoplasms. A familial syndrome? Ann Intern Med 1969; 71: 747-52.

27. Kuchler J, Hartmann W, Waha A, et al. p75NTR induces apoptosis in medulloblastoma cells. Int J Cancer 2011; 128: $1804-12$

28. Jóźwiak J, Sontowska I, Bikowska B, Grajkowska W, Galus R, Roszkowski M. Favourable prognosis in medulloblastoma with extensive nodularity is associated with mitogen-activated protein kinase upregulation. Folia Neuropathol 2011; 49: 257-61.

29. Manning BD, Cantley LC. AKT/PKB signaling: navigating downstream. Cell 2007; 129: 1261-74.

30. Frank AJ, Hernan R, Hollander A, et al. The TP53-ARF tumor suppressor pathway is frequently disrupted in large/cell anaplastic medulloblastoma. Mol Brain Res 2004; 121: 137-40.

31. Gilbertson RJ, Perry RH, Kelly PJ, Pearson AD, Lunec J. Prognostic significance of HER2 and HER4 coexpression in childhood medulloblastoma. Cancer Res 1997; 57: 3272-80.

32. Gajjar A, Hernan R, Kocak M, et al. Clinical, histopathologic, and molecular markers of prognosis: toward a new disease risk stratification system for medulloblastoma. J Clin Oncol 2004; 22: 984-93.

33. Hernan R, Fasheh R, Calabrese $C$, et al. ERBB2 up-regulates S100A4 and several other prometastatic genes in medulloblastoma. Cancer Res 2003; 63: 140-8. 